\title{
Antiproton Acceleration in the Main Injector with RF at Harmonic Number $\mathrm{h}=28$ or $\mathrm{h}=84$
}

\author{
J. A. MacLachlan
}

21 June 02

\begin{abstract}
Antiproton bunches of $1.5 \mathrm{eVs}$ are required for the Tevatron but can not be accelerated through transition in the Main Injector (MI) with the $\mathrm{h}=588 \mathrm{rf}$ system. Chandra Bhat has developed parameters for $\mathrm{h}=28$ acceleration from $8 \mathrm{GeV}$ followed by $\mathrm{h}=588$ acceleration from 25 to $150 \mathrm{GeV}$.[1] This note refines the $\mathrm{h}=28$ scenario and also adapts it to the $\mathrm{h}=84$ case needed for $132 \mathrm{~ns}$ separation. It is practicable to accelerate the $1.5 \mathrm{eVs} \bar{p}$ bunches in about seven seconds with no loss and less than ten percent emittance growth using the existing $2.5 \mathrm{MHz}$ cavities. Both the cycle time and emittance growth are significantly improved by the scheme described in this note. Excellent results can be obtained for the $7.5 \mathrm{MHz}$ case with only $75 \mathrm{kV}$ except that there is a $0.5 \%$ beam loss. For completely clean $53 \mathrm{MHz}$ capture of the tail produced by shape mismatch at transition, $250 \mathrm{kV}$ is desirable for bunch rotation at $26 \mathrm{GeV}$.
\end{abstract}

\section{Outline of the Scheme}

For $\mathrm{h}=28$ the present specification of maximum broadband rf voltage $\hat{V}_{\mathrm{rf}}=2.0 \mathrm{kV}$ in the Recycler (RR) sets the rf level for injection. With the stationary bucket just over a third full, this makes for a comfortable synchronous injection. However, the tune $v_{\mathrm{s}}$ is less than $10^{-4}$, so parameter changes up the parabola must be very slow to avoid unnecessary bunch growth. No systematic minimization of ramp time has been undertaken. An observation that ramp slopes greater than $4 \mathrm{GeV} / \mathrm{s}$ are difficult at more than one point in the cycle is adequate reason to choose this maximum for the entire $\mathrm{h}=28$ fraction of the cycle; there will be only a few of these cycles per store. The cycle length is a consideration primarily for heating of the 2.5 MHz cavities, which may be reduced by slower acceleration if necessary.

The ramp curve is shown in Fig. 1. The first parabola ends at $12 \mathrm{GeV}$ followed by a linear segment to $24 \mathrm{GeV}$ and a parabola onto a front porch at $26 \mathrm{GeV}$ where the handoff to $\mathrm{h}=588$ occurs. The $\dot{p}$ and the rf voltage and phase are kept constant on the linear ramp for $\pm 25 \mathrm{~ms}$ around the time of transition crossing, i. e., a little more than the nonadiabatic time

$$
T_{\mathrm{na}}= \pm\left[\frac{\beta_{T}^{2} \gamma_{T}^{4}}{4 \pi f_{\mathrm{rf}}} \frac{\left|\tan \phi_{\mathrm{s}}\right|}{\dot{\gamma}^{2}}\right]^{1 / 3}
$$

where the symbols are defined and parameter values assigned in Table 1 . The motion on the decreasing parabola is not quite adiabatic; the bunch arrives with a considerable tilt. It is rotated to minimum height at 
low voltage after the $\mathrm{h}=56$ linearizing voltage is turned on; then the $\mathrm{h}=28$ and $\mathrm{h}=56$ voltages are jumped to the maximum $60 \mathrm{kV}$ and $10 \mathrm{kV}$ respectively for a quarter period rotation to minimum width. A matching $3 \mathrm{eVs} \mathrm{h}=588$ bucket $(700 \mathrm{kV})$ is turned on suddenly at time of minimum bunch width. Because the bunch is large, the $\mathrm{h}=588$ portion of the ramp is held to $160 \mathrm{GeV} / \mathrm{s}$ maximum. The $\mathrm{h}=28$ voltage curve is given in Fig. 2(a) and the $h=588$ voltage curve is given in Fig. 2(b). The synchronous phase and the bucket area for the full ramp are shown in Fig. 3. One of the more important changes made to the scheme presented in ref. [1] is raising the front porch energy from 25 to $26 \mathrm{GeV}$ to improve linearity in the matching process.

\section{Results for $h=28$}

The initial $95 \%$ area of the $1.5 \mathrm{eVs}$ bunch is $1.31 \mathrm{eVs}$, and its rms emittance is $0.298 \mathrm{eVs}$; an elliptical distribution is assumed. At $150 \mathrm{GeV}$, the emittances have grown to $1.44(+9.9 \%)$ and $0.308 \mathrm{eVs}(+3.4 \%)$ respectively. The time development of the rms emittance shown in Fig. 4(a) indicates growth after transition and after the switch to $\mathrm{h}=588$ acceleration. In both cases the growth is the result of unavoidable shape mismatch. The $95 \%$ emittance grows by a larger factor because it more strongly reflects the development of a tenuous tail to the distribution by filamentation arising from the small mismatches. These are very promising results; both technical matters and complications in principle arising from the beam current will make it practically impossible to actually match them. Nonetheless, the peak current is reasonable and the complications are generally understood.

\section{Modifications for $h=84$}

The modification to this scheme for $132 \mathrm{~ns}$ bunch spacing is simple in concept but involves some new RR rf. A $2 \mathrm{kV}$ bucket at $7.5 \mathrm{MHz}$ is less than $1.2 \mathrm{eVs}$. To have the same degree of filling used in the $\mathrm{h}=28$ case, the voltage should be $18 \mathrm{kV}$, nine times larger. This is too much for the broadband system, but a resonant cavity of the sort being built for the MI can be included in the RR for shortening the bunches prior to transfer. On the other hand, it is unnecessary to make the $h=84$ voltage in the MI nine times the $60 \mathrm{kV}$ of the $\mathrm{h}=28$ system. The beam can be accelerated to the front porch with no loss and practically no dilution on the same ramp with just under $75 \mathrm{kV}$. However, a rotation at $75 \mathrm{kV}$ does not narrow a $1.5 \mathrm{eVs}$ bunch sufficiently to avoid all later loss. The voltage curves are given in Fig. 5(a) for $\mathrm{h}=84$ and Fig. 5(b) for $\mathrm{h}=588$. The parameters which are specific to the $\mathrm{h}=84$ case are collected in Table 2 .

\section{Results for $h=84$}

Under the somewhat ideal conditions in the modeling, the loss with $75 \mathrm{kV}$ of $\mathrm{h}=84 \mathrm{rf}$ is $0.5 \%$ spread over the $\mathrm{h}=588$ portion of the ramp. The choice of how many $7.5 \mathrm{MHz}$ cavities to build may be set by what is needed by proton coalescing or a judgement on acceptable $\bar{p}$ losses. The considerations of this note favor about $250 \mathrm{kV}$. The ramp is similar to that for the $\mathrm{h}=28$ case except that maximum $\dot{E}$ is reduced to $120 \mathrm{GeV} / \mathrm{s}$. The front porch can be shortened because the bunch manipulation goes a little faster at 7.5 $\mathrm{MHz}$.

Perhaps the solutions for the two cases would have differed more had they been derived completely independently, but for each the results given are satisfactory. The $95 \%$ emittance grows from $1.26 \mathrm{eVs}$ to $1.34 \mathrm{eVs}$ or $6.3 \%$. The rms emittance grows from $0.294 \mathrm{eVs}$ to $0.306 \mathrm{eVs}$ or $4.1 \%$. Figure 4(b) shows 
the growth of the rms emittance of the bunch over the entire cycle. The two sets of results are closely comparable. The smaller emittance growth for the $h=84$ case during the final $h=588$ acceleration suggests a possible gain for the $\mathrm{h}=28$ case by using a slower ramp from 26 to $150 \mathrm{GeV}$.

\section{Effects of Beam Current}

With only $6 \cdot 10^{10} \bar{p}$ 's in a bunch of $1.5 \mathrm{eVs}$, the peak current is much lower than typical for MI operation. However, the accelerating cycle is atypical also. Therefore, a longitudinal impedance consisting of the perfectly conducting wall contribution and a broadband $Z \| / n=2 \mathrm{Ohm}$ was introduced into the optimized $\mathrm{h}=28$ parameter set as a precautionary test. There was no increase in the final $95 \%$ containment area of the bunch. Minor differences were noted including a $0.02 \%$ particle loss spread between $32 \mathrm{GeV}$ and $144 \mathrm{GeV}$. The lost particles were some of the inhabitants of a minor tail to the distribution formed near $8 \mathrm{GeV}$. Check calculations on the first second of the cycle implicate early mismatch rather than general blowup of the bunch. The effect, although practically insignificant, is somewhat curious; it does not appear in the absence of the collective potential or without acceleration in the presence of the collective potential. Thus it appears to be neither a simple bucket shape distortion arising from the beam induced voltage nor an artifact of nonadiabatic initiation of acceleration. However, if the intention were realistic calculations at the $0.02 \%$ level, many refinements would be needed to the model which has been used.

\section{General Results}

1. $1.5 \mathrm{eVs}$ bunches of $6 \cdot 10^{10} \bar{p}$ 's can be accelerated from $8 \mathrm{Gev}$ in the MI without practically important loss or emittance dilution.

2. The existing $60 \mathrm{kV} 2.5 \mathrm{MHz}(\mathrm{h}=28)$ coalescing system in the MI is satisfactory for $36 \times 36$ bunch loading of the Tevatron.

3. Bunches of the same size spaced at $132 \mathrm{~ns}$ can be handled equally well with a $7.5 \mathrm{MHz}(\mathrm{h}=84)$ system.

4. A 7.5 MHz system should be capable of about $250 \mathrm{kV}$.

5. A MI cycle time of $7.5 \mathrm{~s}$ is sufficient for either 2.5 or $7.5 \mathrm{MHz}$ acceleration to $26 \mathrm{GeV}$ followed by $53 \mathrm{MHz}$ acceleration from 26 to $150 \mathrm{GeV}$.

6. No special measures will be required to deal with beam induced voltage other than already planned fundamental rf beam loading compensation.

\section{References}

[1] Chandra Bhat, "Anti-Proton Beam Acceleration in the MI and RF Specifications", Main Injector note MI-0260 (9/21/99) and MI-0260 (rev.) (1/26/00), unpublished 
Table 1: Main Injector rf parameters for $\mathrm{h}=28 \bar{p}$ acceleration. Energy dependent parameters are evaluated at transition energy.

\begin{tabular}{|ll|rl|}
\hline$R_{\mathrm{eq}}$ & mean orbit radius & 528.57 & $\mathrm{~m}$ \\
$\gamma_{T}$ & transition gamma & 20.4 & \\
$E_{\text {inj }}$ & injection energy & 8.93827 & $\mathrm{GeV}$ \\
$E_{\mathrm{max}}$ & top energy & 150.93827 & $\mathrm{GeV}$ \\
$E_{\mathrm{T}}$ & transition energy & 19.14076 & $\mathrm{GeV}$ \\
$\beta_{T}$ & Lorentz $\beta$ at transition & 0.99880 & \\
$\dot{\gamma}$ & maximum rate of change of $\gamma$ & $\hookleftarrow$ & \\
$\hookrightarrow$ & below 26 GeV & 4.263 & $\mathrm{~s}$ \\
$\hookrightarrow$ & 26-150 GeV & 170.526 & $\mathrm{~s}$ \\
$\phi_{\mathrm{S}}$ & synchronous phase & 67.48 & $\mathrm{deg}$ \\
$f_{\text {rf }}$ & accelerating frequency & 2.52449 & $\mathrm{MHz}$ \\
$\hat{V}_{28}$ & maximum 2.5 MHz voltage & 60 & $\mathrm{kV}$ \\
$\hat{V}_{56}$ & maximum 5 MHz voltage (rotation) & 10 & $\mathrm{kV}$ \\
$\hat{V}_{588}$ & maximum 53 MHz voltage & 4 & $\mathrm{MV}$ \\
$\tilde{\varepsilon}_{\text {initial }}$ & initial rms emittance of bunch & 0.298 & $\mathrm{eVs}$ \\
$\tilde{\varepsilon}_{\text {final }}$ & final rms emittance of bunch & 0.308 & $\mathrm{eVs}$ \\
$\varepsilon_{\text {initial }}$ & initial area of 95 \% of bunch & 1.31 & $\mathrm{eVs}$ \\
$\varepsilon_{\text {final }}$ & final area of 95\% of bunch & $\longleftarrow$ & \\
$\hookrightarrow$ & (beam charge ignored) & 1.44 & $\mathrm{eVs}$ \\
$\hookrightarrow$ & (Z & 1.42 & $\mathrm{eVs}$ \\
& total cycle time & 7.345 & $\mathrm{~s}$ \\
\hline
\end{tabular}

Table 2: Main Injector rf parameters specific to $\mathrm{h}=84 \bar{p}$ acceleration only. Energy dependent parameters are evaluated at transition energy.

\begin{tabular}{|ll|rl|}
\hline$\dot{\gamma}$ & maximum rate of change of $\gamma$ & $\hookleftarrow$ & \\
$\hookrightarrow$ & below 26 GeV & 4.263 & $\mathrm{~s}^{-1}$ \\
$\hookrightarrow$ & 26-150 GeV & 127.895 & $\mathrm{~s}^{-1}$ \\
$\phi_{\mathrm{s}}$ & synchronous phase & 55.79 & $\mathrm{deg}$ \\
$f_{\mathrm{rf}}$ & accelerating frequency & 7.54378 & $\mathrm{MHz}$ \\
$\hat{V}_{84}$ & maximum 7.5 MHz voltage & $\hookleftarrow$ & \\
$\hookrightarrow$ & (acceleration) & 75 & $\mathrm{kV}$ \\
$\hookrightarrow$ & (rotation) & 270 & $\mathrm{kV}$ \\
$\hat{V}_{168}$ & maximum 15 MHz voltage (rotation) & 45 & $\mathrm{kV}$ \\
$\varepsilon_{\text {initial }}$ & initial area of 95 \% of bunch & 1.26 & $\mathrm{eVs}$ \\
$\varepsilon_{\text {final }}$ & final area of 95 \% of bunch & 1.34 & $\mathrm{eVs}$ \\
& total cycle time & 7.516 & $\mathrm{~s}$ \\
\hline
\end{tabular}




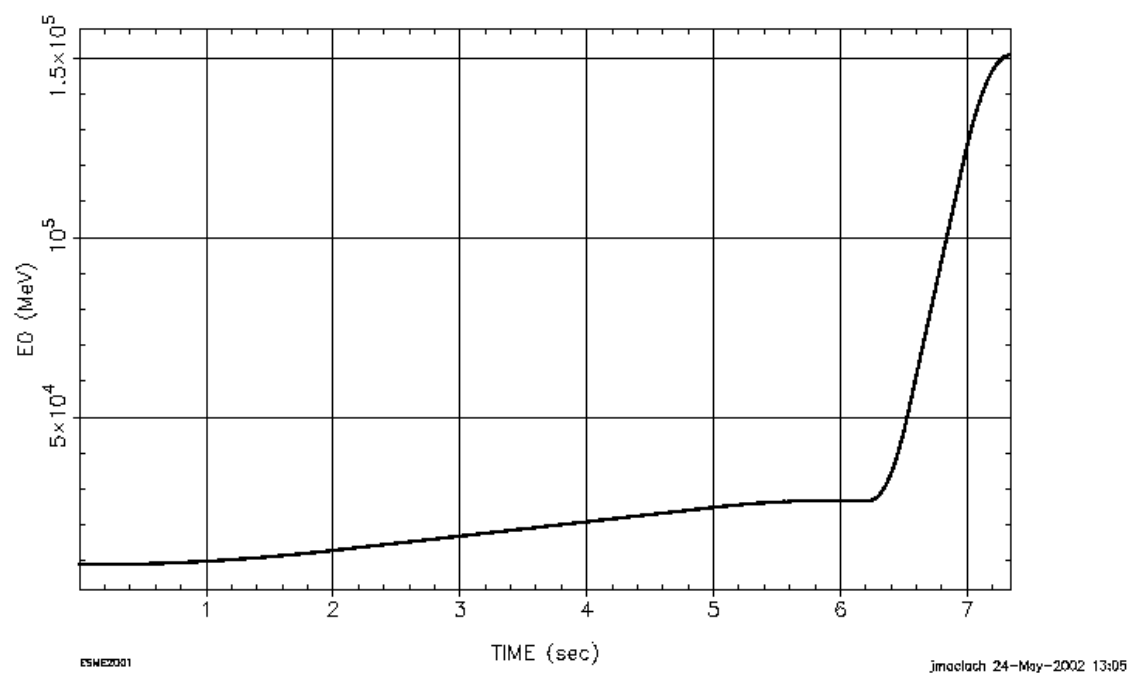

Figure 1: Ramp (energy $v s$. time) for a full $\bar{p}$ cycle with $\mathrm{h}=28$ acceleration to $26 \mathrm{GeV}$



(a) $h=28$ voltage $(8-26 \mathrm{GeV})$



(b) $\mathrm{h}=588$ voltage $(26-150 \mathrm{GeV})$

Figure 2: Voltage curves for $\bar{p}$ acceleration using $\mathrm{h}=28$ to $26 \mathrm{GeV}$

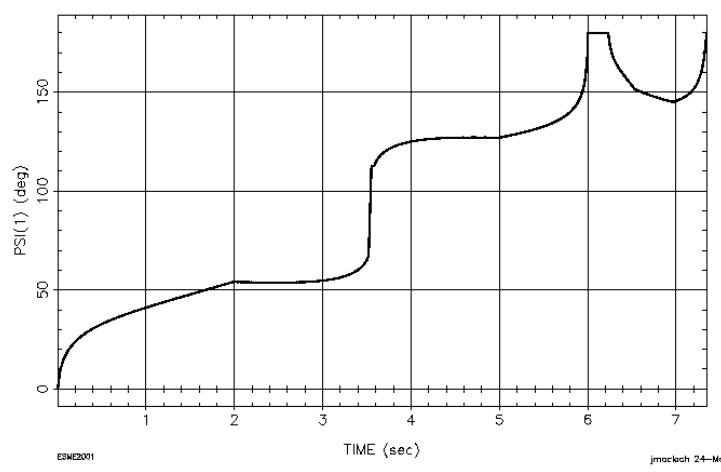

(a) Synchronous phase - both $\mathrm{h}=28$ and $\mathrm{h}=588$

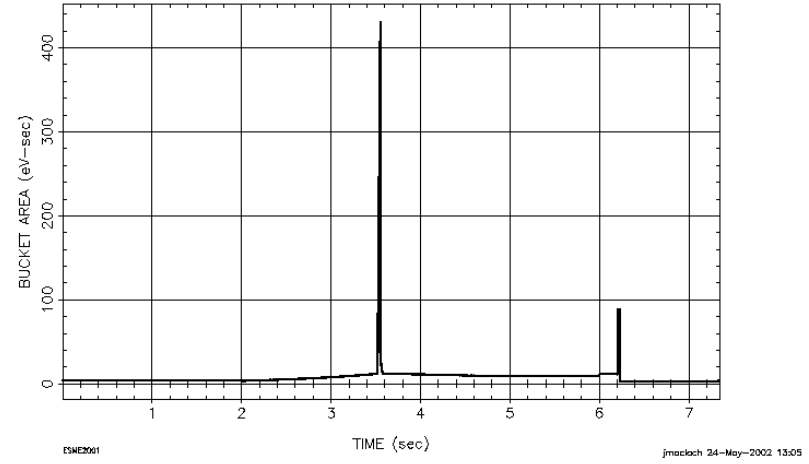

(b) Bucket area for entire cycle - values near $3.55 \mathrm{~s}$ (transition) not meaningful

Figure 3: Parameter curves for $\bar{p}$ acceleration using $\mathrm{h}=28$ to $26 \mathrm{GeV}$ 




(a) Using $\mathrm{h}=28$ acceleration to $26 \mathrm{GeV}$



(b) Using $\mathrm{h}=84$ acceleration to $26 \mathrm{GeV}$

Figure 4: $\varepsilon_{\text {rms }} v s$. time using low harmonic number acceleration to $26 \mathrm{GeV}$



(a) For $\mathrm{h}=84$ acceleration $(8-26 \mathrm{GeV})$

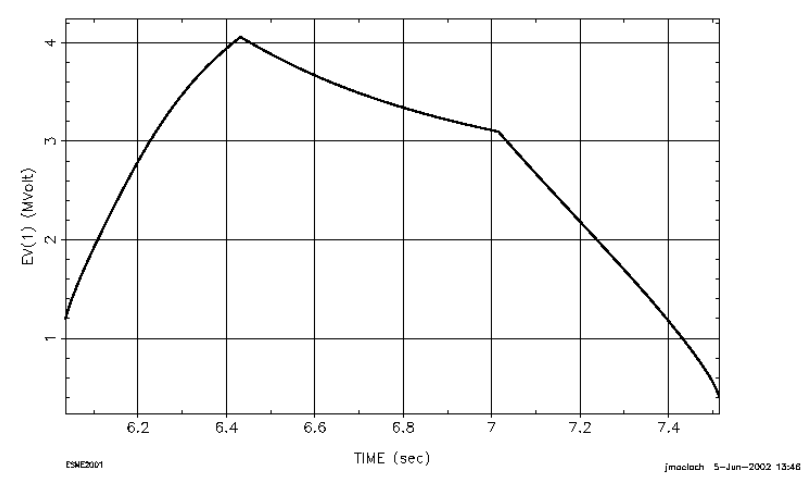

(b) For $\mathrm{h}=588$ acceleration $(26-150 \mathrm{GeV})$

Figure 5: Voltage curves for $\bar{p}$ acceleration from 8 to $150 \mathrm{GeV}$ with $\mathrm{h}=84$ to $26 \mathrm{GeV}$ 